\title{
INDEPENDENT ON-ORBIT GEOMETRIC CALIBRATION OF OPTICAL SATELLITE BASED ON WEIGHTED VIRTUAL OBSERVATION
}

\author{
Yang $\mathrm{Bo}^{1}$, Pi Yingdong ${ }^{2 *}$ \\ ${ }^{1}$ State Key Laboratory of Information Engineering in Surveying, Mapping and Remote Sensing, Collaborative \\ Innovation Center of Geospatial Technology, Wuhan University, Wuhan China - 93341186@qq.com \\ ${ }^{2}$ School of Remote Sensing and Information Engineering, Wuhan University, Wuhan, China - \\ piyingdong@whu.edu.cn
}

\section{Commission I, WG I/4}

KEY WORDS: Optical satellite, Independent geometric calibration, Mutual constraints, Images combinations, Weight virtual observation

\begin{abstract}
:
On-orbit geometric calibration is an essential technology to improve the initial geometric accuracy of the optical satellite images. The presented methods usually take the absolute constraint from calibration site data into the imaging model to deduce exact systematic error parameters. However, the characteristic of strong dependence on the high-accuracy calibration site data leads to limitations such as high cost and poor efficiency of these conventional methods. In order to make better use of the mutual constraints among the images, and to get rid of the dependence of calibration site, we conduct an investigation on the on-orbit geometric calibration using the mutual constraints of overlapped images instead of absolute constraint from calibration site for optical satellite. To ensure the stable solution of calibration equation, a weighted virtual observation is introduced into calibration model to improve its condition. The method is verified with a pair of simulated images, and the satisfactory results indicate that the method is effective and reasonable.
\end{abstract}

\section{INTRODUCTION}

Geometric calibration of the optical satellite can be divided into two categories according to the different commissioning stages: laboratory calibration and on-orbit geometric calibration. The former is always undertaken in the laboratory using precision optical instruments such as parallel light tubes before the satellite launch. However, the camera parameters usually change to a certain extent due to complex factors such as the vibration in the process of satellite launching, stress release and the change in imaging environment. Therefore, the geometric calibration should be re-performed on-orbit to improve the initial geometric accuracy of the imaging model.

Conventional on-orbit geometric calibration method usually use the absolute constraints identified from reference data covering the ground calibration site to retrieve the physical camera parameters or the fitting model (Mulawa, 2004; Wang et al., 2014).

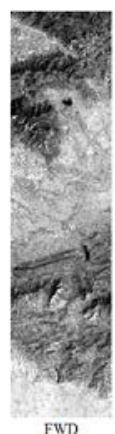

(a)

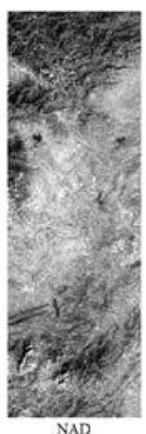

NAD

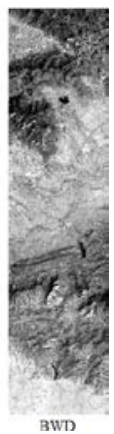

BWD

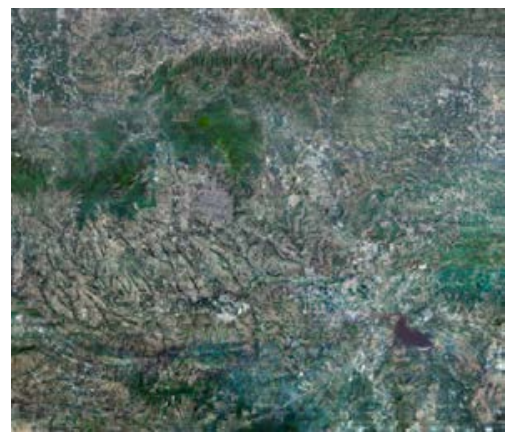

(b) Reference DOM.

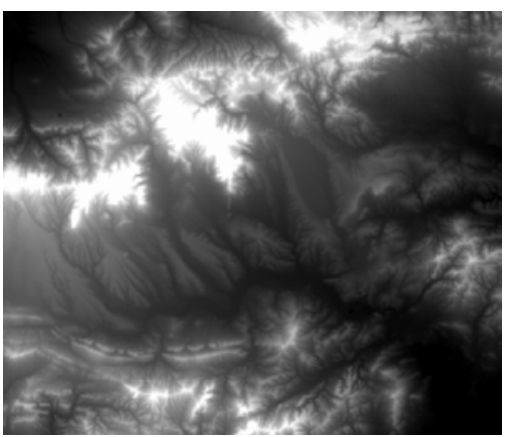

(c) Reference DSM.

Figure 1. Essential data used for geometric calibration

\footnotetext{
Corresponding author
} 
satellite was performed by Wuhan university and CRESDA. Referring to Figure 1, the used reference data is the digital ortho map (DOM) and digital surface model (DSM) covering SongShan calibration site. After the cloud-free TLC images (Figure 1(a)) over the area were collected, the geometric calibration was performed, in which the camera parameters were fitted using a viewing-angle model, and a step-wise estimate was adopted aiming to the stable solution of calibration parameters. After calibration, the geo-positioning accuracy and internal accuracy of TLC imagery were improved to about $15 \mathrm{~m}$ and better than 1 pixel respectively (Cao et al., 2015; Chen et al., 2015; Yang et al., 2017).

However, this approach may appear to be easy executed on the surface, but suffers to critical limitation due to the strong dependence on the precise calibration site data. Since the reference data is always produced by aerial surveying under the constraint of many manual measured ground control points (GCPs), so that the generation and maintenance of the available reference data is a process of high cost and low time. In addition, the long return visit cycle make it difficult to obtain the cloud-free images over calibration site, which further reduces the timeliness of geometric calibration (Pi et al., 2017).

Aiming to the limitations of conventional method, this paper describes a new method for on-orbit geometric calibration of optical satellite. In the method, the relative constraint among overlapped images instead of absolute constraint from GCPs is used to deduce the optimal systematic parameters. Therefore, this new method can get rid of the limitations of conventional method stemming from the strong dependence on calibration site.

A great challenge need to conquer in this method is the unstable estimate of the calibration parameters. First, the relative constrain condition is useful for high order error, but it cannot be used to restrain the translation error existing in internal parameters. Second, the strong correlation between the elevation error and the internal accuracy will result in the rank-defect of solution model. We developed some improvement to solve both problems. For first problem, we introduced a weighted virtual observation to improve calibration model. For the second problem, we draw lessons from the idea of using a DEM as an observable for orientation and positioning proposed by Ebner Heinrich in 1991 (Ebner et al. 1991), and applied an elevation constraint to conquer the strong correlation.

\section{DESCRIPTION OF THE METHOD}

We take the linear optical satellite sensor as an example to illustrate this new method, and the planar array sensor can be regarded as an extension in the direction of column. The method is described in following three aspects.

\subsection{Effect analysis of the relative constraint}

If we want to achieve the independent on-orbit geometric calibration with the relative constraints among images, at least two overlapped images should be collected, and the overlap in the direction along CCD needs to be greater than $50 \%$. Thus, the mutual constraint between pre and second half of the camera distortion can be realized, and the whole CCD range is covered. Referring to Figure 2(a), under the effect of mutual constraint of corresponding points identified from overlap images, the camera distortion reaches optimal condition when the statistics of relative geometric residual of the corresponding point is the smallest. For a non-periodic camera internal distortion, only when the high order distortion is compensated, this optimal condition can be achieved (Figure 2(b)). However, the translation error (principal point error) in internal distortion is independent with geometric residual of the corresponding point. Thus, this error cannot be corrected in this method, and the estimate will be unstable if it is solved directly in this method. Aiming to this problem, we mean to introduce into some prior position constraint to improve the calibration model (Figure 2(c)). The details of this part will be detailed individually in next section.

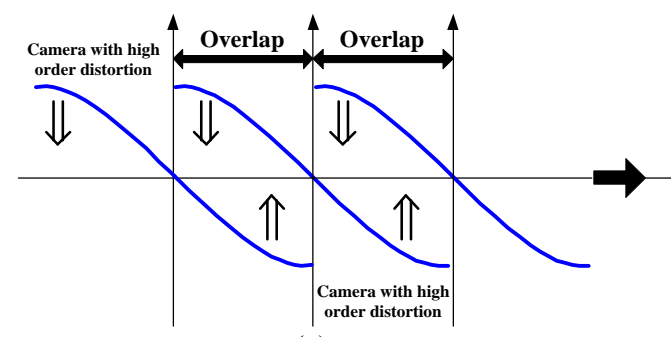

(a)

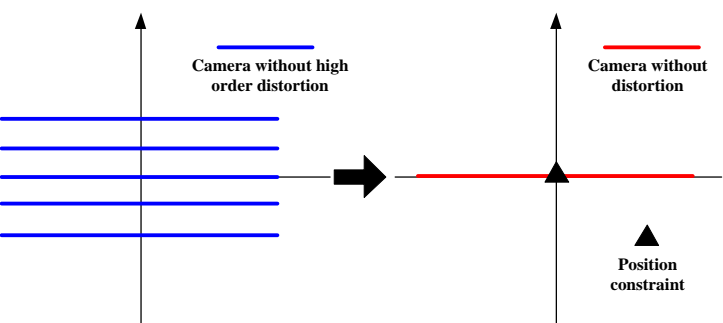

(b) (c)

Figure 2. Description of auto internal calibration using mutual constraint of overlapped images

In addition, if the method is used in the calibration of a planar array sensor, the mutual constraint along the linear CCD should be extended to along two directions of the planar array CCD. In practical application, four images of more than $50 \%$ overlap in both directions are appropriate for providing the mutual constraints needed in this method. 


\subsection{Introduction of weighted virtual observation}

To conquer the unstable estimate caused by the translation error in integral solution, we mean to introduce into some prior position constraint to improve the calibration model. This constraint can fix the position of camera distortion in the camera coordinate system, but it will not affect the correction of high order distortion. For the rigorous camera model, the translation error can be directly ignored and only calibrate the focal length and optical distortion. But the rigorous camera model may suffer some problems such as under fitting and strong correlation, so that it is rarely used in the practical geometric process of optical satellite. We usually use a viewing-angle model to replace the rigorous camera model (Pi et al., 2017; Wang et al., 2017; Yang et al., 2017).

$$
V_{\text {cam }}^{T}=\left(\frac{x}{z}, \frac{y}{z}, 1\right)=\left(\tan \left(\psi_{x}\right), \tan \left(\psi_{y}\right), 1\right)
$$

The three-order fitted viewing-angle models for linear sensor and planar array sensor are shown as followings:

$$
\begin{gathered}
\left\{\begin{array}{l}
\tan \left(\psi_{x}\right)=a_{0}+a_{1} s+a_{2} s^{2}+a_{3} s^{3} \\
\tan \left(\psi_{y}\right)=b_{0}+b_{1} s+b_{2} s^{2}+b_{3} s^{3}
\end{array}\right. \\
\left\{\begin{array}{c}
\tan \left(\psi_{x}\right)=a_{0}+a_{1} s+a_{2} l+a_{3} s l+a_{4} s^{2} \\
+a_{5} l^{2}+a_{6} s^{2} l+a_{7} s l^{2}+a_{8} s^{3}+a_{9} l^{3} \\
\tan \left(\psi_{y}\right)=b_{0}+b_{1} s+b_{2} l+b_{3} s l+b_{4} s^{2} \\
+b_{5} l^{2}+b_{6} s^{2} l+b_{7} s l^{2}+b_{8} s^{3}+b_{9} l^{3}
\end{array}\right.
\end{gathered}
$$

where, $S$ in (2) is the CCD detector numbers, $(s, l)$ in (3) is the detector's image plane coordinate.

For the viewing-angle model, we restrain this translation error by introducing virtual control points (VCPs) into the calibration model as weighted observations. VCP is generated on a constant elevation plane using the initial imaging model (Figure 3). VCP is used as real GCP in the solution equation, thus an appropriate weight is important to ensure estimate convergence and accuracy of the calibration.

As shown in Figure 3, the pixels of each row in the raster image are one-to-one correspondence to the CCD detectors. Thus the image can be adjusted is divided into regular grids according to this raster. The center of each grid is defined by $p_{i}$, and the corresponding object space point $P_{i}$ can be determined by intersection using the initial imagine parameters with an appropriate elevation datum. For this case, the imagery point $p_{i}$ and the object space point $P_{i}$ constitute a set of VCPs.
But it is important to note that the VCP should be generated using the same CCD detector to avoid impact on the internal accuracy. Thus, at most a VCP can be generated at the same imagery pixel in each line for linear image, while one image can produce only one VCP for array image.

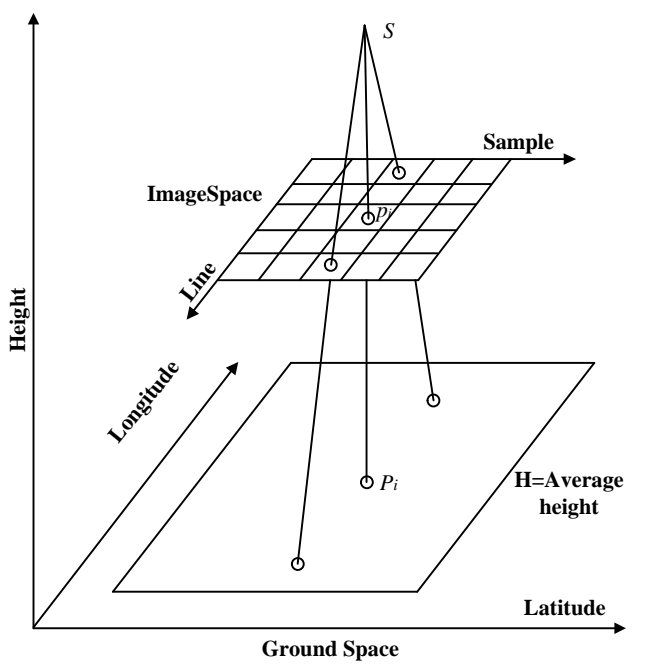

Figure 3. Generation of virtual control points for linear satellite image

\subsection{DEM aided stable estimation}

Another problem need to be solved is the correlation between the internal parameters and elevation. If both kinds of parameters are unknown in calibration solution, the solution equation is rank-defect, so that the stable estimation cannot be achieved. To solve this problem, an additional constraint was introduced into the calibration model by interpolating the elevation from the reference DSM and regarding it as the true elevation of a corresponding point.

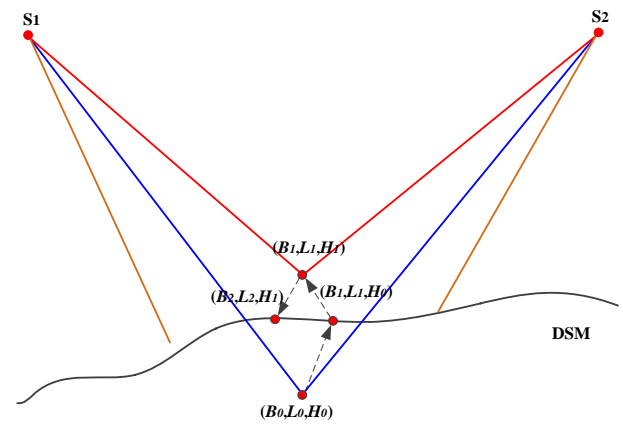

Figure 4. Elevation interpolation from a reference

DSM.

Referring to the Figure 4, the DSM provides the elevation of ground points as follows. First, an iteration process is used to calculate the initial ground planimetric coordinates of corresponding point, since the ground locations resulting from the corresponding point on different images may be different, thus we take the average position $\left(B_{0}, L_{0}\right)$ of corresponding 
ground points as the initial position of this point pair. Second, the initial elevation $H_{0}$ can be interpolated from the reference DSM according to planimetric coordinates $\left(B_{0}, L_{0}\right)$. Third, we use interpolated elevation $H_{0}$ as the true elevation value for the next iteration of the solution, and update the elevation according to the calculated planimetric coordinates during the solution iteration.

\section{EXPERIMENT AND DISCUSSION}

\subsection{Description of dataset}

To explain the feasibility of the method from the essence of mathematics, we performed the experiment using a set of simulated images. Thus, the calibration accuracy can be verified accurately by comparing the estimated camera parameters with the designed parameters. We used the real orbit data of ZY-3 satellite to simulated calibration images. The camera was designed as a center FOV camera, and its physical parameters are listed in Table 1.

\begin{tabular}{|l|c|}
\hline Item & The designed value \\
\hline Focal length & $2.5(\mathrm{~m})$ \\
CCD detector number & 6000 \\
Ground distance sample & $1.0(\mathrm{~m})$ \\
CCD detector size & $5.0(\mu \mathrm{m})$ \\
Orbit height & $505(\mathrm{~km})$ \\
\hline
\end{tabular}

Table 1 . The designed physical parameters

With the designed parameters, the initial viewing-angle coefficients which can be used as the criteria for accuracy evaluation can be determined. Then, the overlapped images with an overlap of 50\% were generated under the support of precise DOM and DSM covering SongShan. The images are simulated according to the rigorous imaging process of the satellite, as shown in Figure 5.
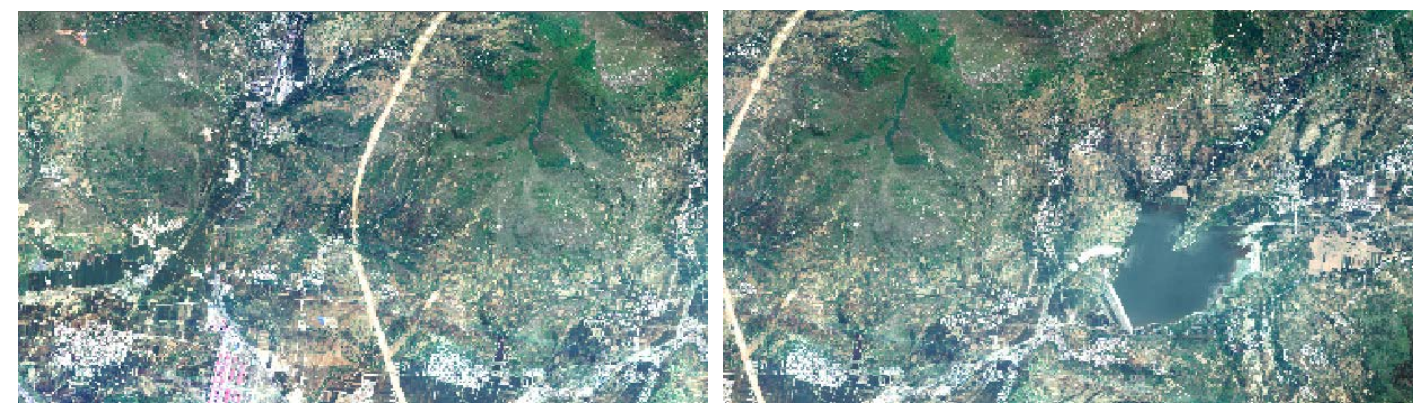

Figure 5. Overlapped images with identified corresponding point

To utilize the cross constraint correlation of the simulated image pair, a mature matching algorithm based on the least squares method was applied to identify the corresponding imagery points. A totally of 1758 corresponding points were identified from the images, and 3 VCPs at the same CCD detector (center detector) for an image, thus a total of 6 VCPs were generated. With the these corresponding points, VCPs, and an aided DSM, the internal calibration can be achieved in the proposed method.

\subsection{Calibration result}

Three sets of different initial internal distortions were designed to illustrate the universality of this method. These distortions can be described as $\sin x, \cos x$ and sqrtx according to the corresponding mathematical model. We extend initial distortion cross the CCD to as large as 10 pixels, and 2 pixels along the CCD at the both ends of the CCD by changing the detector size. The shapes of these distortions are shown in Figure 6.

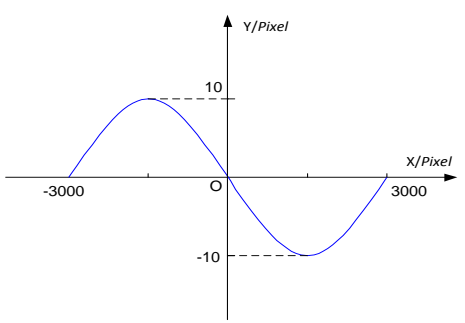

(a) $\sin x$

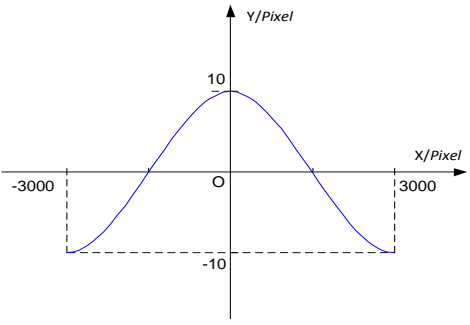

(b) $\cos x$

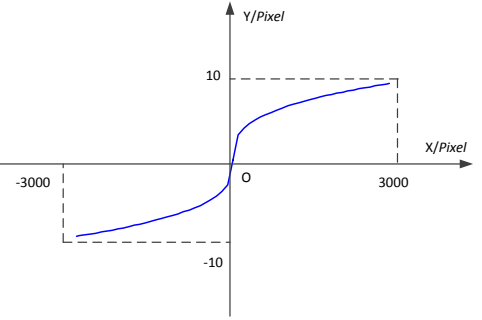

(c) sqrtx

Figure 6. The designed internal distortions with different shapes 


\begin{tabular}{|c|c|c|c|c|c|c|c|c|}
\hline Distortion & $a_{0}$ & $a_{1}$ & $a_{2}$ & $a_{3}$ & $b_{0}$ & $b_{1}$ & $b_{2}$ & $b_{3}$ \\
\hline $\operatorname{Sin} x$ & $\begin{array}{c}-8.811 \mathrm{E}- \\
06\end{array}$ & $\begin{array}{c}-1.426 \mathrm{E} \\
-08\end{array}$ & $\begin{array}{c}8.611 \mathrm{E}- \\
12\end{array}$ & $\begin{array}{c}-9.580 \mathrm{E} \\
-16\end{array}$ & $\begin{array}{c}-5.995928 \\
\text { E-03 }\end{array}$ & $\begin{array}{c}1.999928 \\
\text { E-06 }\end{array}$ & $\begin{array}{c}2.398 \mathrm{E}-1 \\
4\end{array}$ & $\begin{array}{c}-2.667 \mathrm{E}- \\
18\end{array}$ \\
\hline $\operatorname{Cos} x$ & $\begin{array}{c}1.461 \mathrm{E}- \\
06\end{array}$ & $\begin{array}{c}-3.327 \mathrm{E} \\
-08\end{array}$ & $\begin{array}{c}2.145 \mathrm{E}- \\
11\end{array}$ & $\begin{array}{c}-2.947 \mathrm{E} \\
-15\end{array}$ & $\begin{array}{c}-5.995928 \\
\text { E-03 }\end{array}$ & $\begin{array}{c}1.999928 \\
\text { E-06 }\end{array}$ & $\begin{array}{c}2.398 \mathrm{E}-1 \\
4\end{array}$ & $\begin{array}{c}-2.667 \mathrm{E}- \\
18\end{array}$ \\
\hline Sqrtx & $\begin{array}{c}-2.698 \mathrm{E}- \\
05\end{array}$ & $\begin{array}{c}1.399 \mathrm{E}- \\
08\end{array}$ & $\begin{array}{c}-2.491 \mathrm{E}- \\
12\end{array}$ & $\begin{array}{c}2.765 \mathrm{E}- \\
16\end{array}$ & $\begin{array}{c}-5.995928 \\
\text { E-03 }\end{array}$ & $\begin{array}{c}1.999928 \\
\text { E-06 }\end{array}$ & $\begin{array}{c}2.398 \mathrm{E}-1 \\
4\end{array}$ & $\begin{array}{c}-2.667 \mathrm{E}- \\
18\end{array}$ \\
\hline
\end{tabular}

Table 2. Initial internal parameters for different distortions

The initial internal parameters were used as the initial values of internal parameters in calibration solution. The least square method was used to achieve the optimal estimation of calibration parameters. With corresponding points and VCPs, the calibration equation was established, and then the optimal estimation was therefore achieved under the restrain of a DSM. The calculation for three distortions all were achieved within two iterations. The calibration results are listed in Table 3.

\begin{tabular}{|c|c|c|c|c|c|c|c|c|}
\hline Distortion & $a_{0}$ & $a_{1}$ & $a_{2}$ & $a_{3}$ & $b_{0}$ & $b_{1}$ & $b_{2}$ & $b_{3}$ \\
\hline True Value & 0.0000 & 0.0000 & 0.0000 & 0.0000 & $\begin{array}{c}-5.999928 \\
\text { E-03 }\end{array}$ & $\begin{array}{c}1.999928 \\
\text { E-06 }\end{array}$ & $\begin{array}{c}2.400 \mathrm{E}-1 \\
4\end{array}$ & $\begin{array}{c}-2.667 \mathrm{E}- \\
18\end{array}$ \\
\hline $\operatorname{Sin} x$ & $\begin{array}{c}1.114 \mathrm{E}- \\
07\end{array}$ & $\begin{array}{c}-1.459 \mathrm{E}-1 \\
0\end{array}$ & $\begin{array}{c}\text { 5.983E- } \\
14\end{array}$ & $\begin{array}{c}-6.681 \mathrm{E} \\
-18\end{array}$ & $\begin{array}{c}-5.995929 \\
\text { E-03 }\end{array}$ & $\begin{array}{c}1.999928 \\
\text { E-06 }\end{array}$ & $\begin{array}{c}2.391 \mathrm{E}-1 \\
4\end{array}$ & $\begin{array}{c}-2.653 \mathrm{E}- \\
18\end{array}$ \\
\hline $\operatorname{Cos} x$ & $\begin{array}{c}1.527 \mathrm{E}- \\
05\end{array}$ & $\begin{array}{c}-1.376 \mathrm{E}-1 \\
0\end{array}$ & $\begin{array}{c}5.623 \mathrm{E}- \\
14\end{array}$ & $\begin{array}{c}-6.228 \mathrm{E} \\
-18\end{array}$ & $\begin{array}{c}-5.995930 \\
\text { E-03 }\end{array}$ & $\begin{array}{c}1.999929 \\
\text { E-06 }\end{array}$ & $\begin{array}{c}2.380 \mathrm{E}-1 \\
4\end{array}$ & $\begin{array}{c}-2.648 \mathrm{E}- \\
18\end{array}$ \\
\hline Sqrtx & $\begin{array}{c}9.790 \mathrm{E}- \\
08\end{array}$ & $\begin{array}{c}-1.454 \mathrm{E}-1 \\
0\end{array}$ & $\begin{array}{c}6.005 \mathrm{E}- \\
14\end{array}$ & $\begin{array}{c}-6.743 \mathrm{E} \\
-18\end{array}$ & $\begin{array}{c}-5.995929 \\
\text { E-03 }\end{array}$ & $\begin{array}{c}1.999928 \\
\text { E-06 }\end{array}$ & $\begin{array}{c}2.395 \mathrm{E}-1 \\
4\end{array}$ & $\begin{array}{c}-2.658 \mathrm{E}- \\
18\end{array}$ \\
\hline
\end{tabular}

Table 3. Estimated internal parameters for different distortions

\subsection{Accuracy assessment}

The calibration accuracy can be evaluated by comparing the calculated and true viewing-angle of each CCD detector. However, even though the applied VCPs can ensure the stable solution of calibration model, the initial translation error is also preserved by the VCPs. Considering the internal translation error can be compensated perfectly by external parameters, it is reasonable to only calibrate high order error in internal calibration. But the possible disturb of remaining constant errors should be excluded when we assess accuracy using estimated viewing-angles.

The remaining constant errors were excluded by subtracting the mean value of all detector viewing-angles. Therefore, we could calculate the high order residual of view-angle for all CCD detectors based on the estimated and the true designed internal parameters. The root mean square (RMS), mean value (Mean) and maximum value (Max) of these residuals were counted. The statistical results are listed in Table 4.

\begin{tabular}{|l|c|c|c|c|c|c|c|}
\hline \multirow{2}{*}{ Distortion } & \multicolumn{3}{|c|}{ RMS } & \multicolumn{2}{c|}{ Mean } & \multicolumn{2}{c|}{ Maximum } \\
\cline { 2 - 7 } & RMS_X & RMS_Y & RMS_XY & Mean_X & Mean_Y & Max_X & Max_Y \\
\hline Unit & arcsec & arcsec & arcsec & arcsec & arcsec & arcsec & arcsec \\
Sinx & 0.000093 & 0.005758 & 0.005759 & 0.000000 & 0.000000 & 0.000223 & 0.017332 \\
Cosx & 0.000150 & 0.005295 & 0.005297 & 0.000000 & 0.000000 & 0.000335 & 0.015321 \\
Sqrtx & 0.000087 & 0.005836 & 0.005836 & 0.000000 & 0.000000 & 0.000198 & 0.018039 \\
\hline
\end{tabular}

Table 4. Calibration accuracy for different internal distortions

As shown in Table 4, the comprehensive calibration accuracy better than 0.006 arcsec was achieved for three distortions according to RMS. As a pixel is equivalent to 0.4 arcsec according to the designed 
physical parameters, the presented method can achieve accuracy about 0.015 pixels for designed camera. Additionally, the difference between Mean value and Maximum is so small that the geometric consistency of the calibration result is also excellent, which indicates the presented method is effective on the inversion of high order (one order and more) internal errors, and the presented method is reasonable

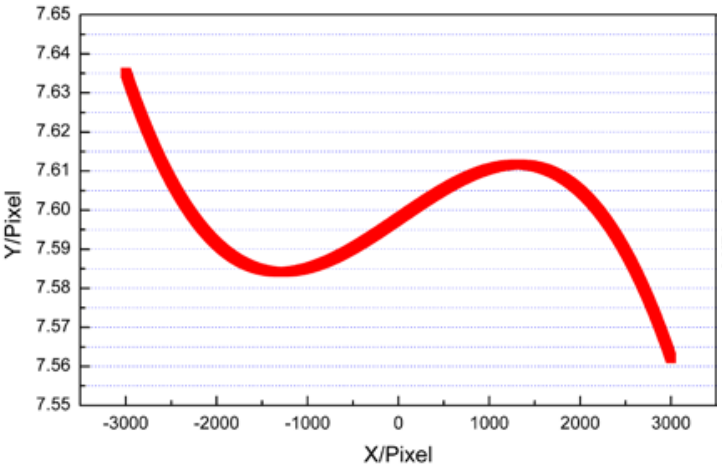

(a) Estimated distortion error curve and available on the principle of mathematics.

To show the satisfactory calibration result more obviously, we drawn the internal distortion curves based on the estimated calibration parameters. Taking the calibration parameters estimated based on initial distortion Cosx as an example, the distortion curves are shown in Figure 7.

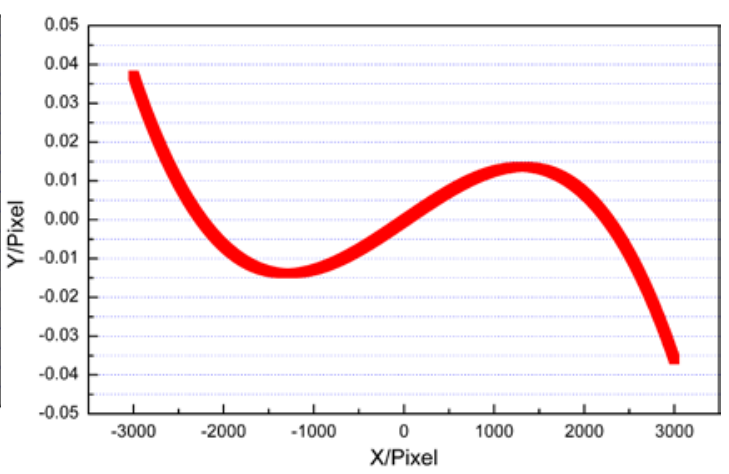

(b) Distortion curve that exclude translation error

Figure 7. The distortion curves of estimated internal parameters

It can be observed that the internal parameters still include internal distortion as high as 7.5 pixels after geometric calibration, but the remaining distortion is mainly translation error, whereas the high order internal distortion have been compensated perfectly. As shown in Figure 7(b), when the remaining translation error is excluded, the internal distortion is improved to better than 0.04 pixels. Thus, the presented method can effectively compensate the high order internal distortion error while ensuring the robust solution.

\section{CONCLUSION WITH SUMMARY}

This paper proposes a new method aiming to the independent geometric calibration of optical satellite, in which the relative constraint among overlapped images instead of GCPs is used to deduce calibration parameters. To ensure the stable estimation of calibration, a novel weighted virtual observation is introduced to improve the condition of calibration model, and a DSM is used as elevation constraint to conquer the correlation between parameters. In the experiment, the method is conducted on a pair of simulated images, the excellent convergence of the solution and the satisfactory accuracy indicate the method is effective and reasonable. With this method, we don't need to use the absolute constraint of GCPs. Therefore, we can get rid of the limitations of conventional method due to the dependence on the high cost calibration site.

\section{ACKNOWLEDGEMENTS}

The authors would like to thank the editors and anonymous reviewers for their valuable comments, which helped improve this manuscript. This work was substantially supported by the National Natural Science Foundation of China (Project Nos. 41601492 and 91638201) and the National Key Research and Development Program of China (Project Nos. 2016YFB0501402). These supports are valuable.

\section{REFERENCES}

Cao, J. S., Yuan, X. X., et al, 2015. In-orbit Geometric Calibration and Validation of ZY-3 Three-line

Cameras Based on CCD-Detector Look Angles. The

Photogrammetric Recor. 30(150), pp. 211-226.

Chen, Y., Xie, Z., et al, 2015. Calibration and Validation of ZY-3 Optical Sensors. IEEE Transactions on Geoscience and Remote Sensing. 53(8), pp. 4616-4626.

Ebner, H., Strunz, G., and Colomina, I., 1991. Block triangulation with aerial and space imagery using DTM as control information. In: ACSM-ASPRS Annual Convention, Technical Papers, Baltimore, vol.5, pp. 76-85.

Mulawa, D., 2004. On-Orbit Geometric Calibration of the Orb-View3 High-Resolution Imaging Satellite. In: ISPRS XXth Congress Proceedings. Istanbul, The International Archives of the Photogrammetry, Remote Sensing and Spatial Information Sciences, Vol. XXXV-B1, pp. 1-7. 
The International Archives of the Photogrammetry, Remote Sensing and Spatial Information Sciences, Volume XLII-1, 2018 ISPRS TC I Mid-term Symposium “Innovative Sensing - From Sensors to Methods and Applications", 10-12 October 2018, Karlsruhe, Germany

Pi, Y. D., Yang, B., Wang, M., et al, 2017. On-orbit geometric calibration using a cross-image pair for the linear sensor aboard the agile optical satellite. IEEE Geoscience \& Remote Sensing Letters. 14(7), pp. 1-5.

Wang, M., Yang, B., Hu, F., et al, 2014. On-Orbit Geometric Calibration Model and Its Applications for High-Resolution Optical Satellite Imagery. Remote Sensing. 6(5), pp. 4391-4408.

Wang, M., Cheng, Y., Chang, X., et al, 2017. On-orbit geometric calibration and geometric quality assessment for the high-resolution geostationary optical satellite GaoFen4. Isprs Journal of Photogrammetry \& Remote Sensing. 125, pp. 63-77.

Yang B, Wang M, Xu W, et al, 2017. Large-scale block adjustment without use of ground control points based on the compensation of geometric calibration for ZY-3 images. Isprs Journal of Photogrammetry \& Remote Sensing, 134, pp. 1-14. 